\title{
Zinc sulfate as an adjunct to methylphenidate for the treatment of attention deficit hyperactivity disorder in children: A double blind and randomized trial [ISRCTN64I3237I]
}

\author{
Shahin Akhondzadeh*1,2, Mohammad-Reza Mohammadi ${ }^{1}$ and \\ Mojgan Khademi ${ }^{1}$
}

\author{
Address: ${ }^{1}$ Pychiatric Research Centre, Roozbeh Hospital, Tehran University of Medical Sciences, South Kargar Street, Tehran 13185, Iran and \\ ${ }^{2}$ Institute of Medicinal Plants, Tehran, Iran \\ Email: Shahin Akhondzadeh* - s.akhond@neda.net; Mohammad-Reza Mohammadi - mrmohammadi@yahoo.com; \\ Mojgan Khademi - a3057@sina.tums.ac.ir \\ * Corresponding author
}

This article is available from: http://www.biomedcentral.com/l47I-244X/4/9

(C) 2004 Akhondzadeh et al; licensee BioMed Central Ltd. This is an Open Access article: verbatim copying and redistribution of this article are permitted in all media for any purpose, provided this notice is preserved along with the article's original URL.

\begin{abstract}
Background: Attention-deficit hyperactivity disorder is an early-onset, clinically heterogenous disorder of inattention, hyperactivity, and impulsiveness. The diagnosis and treatment of attention-deficit hyperactivity disorder continues to raise controversy, and, there is also an increase in treatment options. In this 6-week double blind, placebo controlled-trial, we assessed the effects of zinc plus methylphenidate in the treatment of children with attention deficit hyperactivity disorder. To the best of our knowledge, this study is the first double blind and placebo controlled clinical trial assessing the adjunctive role of zinc in ADHD.

Methods: Our subjects were 44 outpatient children ( 26 boys and 18 girls) between the ages of $5-11$ (mean \pm SD was $7.88 \pm 1.67$ ) who clearly met the DSM IV diagnostic criteria for attention-deficit hyperactivity disorder and they were randomized to methylphenidate $1 \mathrm{mg} / \mathrm{kg} /$ day + zinc sulfate $55 \mathrm{mg} /$ day (with approximately $15 \mathrm{mg}$ zinc element) (group I) and methylphenidate I mg/kg/day + placebo (sucrose $55 \mathrm{mg}$ ) (group 2) for a 6 week double blind clinical trial. The principal measure of the outcome was the Teacher and Parent ADHD Rating Scale. Patients were assessed by a child psychiatrist at baseline, 14,28 and 42 days after the medication started.

Results: The present study shows the Parent and Teacher Rating Scale scores improved with zinc sulfate over this 6-week, double blind and placebo controlled trial. The behavior of the two treatments was not homogeneous across the time. The difference between the two protocols was significant as indicated by the effect on the group, the between-subjects factor $(F=4.15$, d.f. $=I, P=0.04 ; F=4.50$, d.f. $=I, P=0.04$ respectively). The difference between the two groups in the frequency of side effects was not significant.

Conclusions: This double-blind, placebo-controlled study demonstrated that zinc as a supplementary medication might be beneficial in the treatment of children with attention-deficit hyperactivity disorder. However, further investigations and different doses of zinc are required to replicate these findings in children with ADHD.
\end{abstract}




\section{Background}

Attention-deficit/hyperactivity disorder (ADHD) is the most common behavioral disorder of childhood and is recognized as one of the most prevalent chronic health conditions in school-aged children, affecting an estimated $3 \%$ to $5 \%[1,2]$. The key features of ADHD include hyperactivity, impulsiveness, and inattention [1,2]. Although its etiology remains unknown, data from family genetic, twin and adoption studies as well as segregation analysis suggest a genetic origin for some forms of this disorder $[1,2]$. Successful management of ADHD relies heavily on the accuracy of its diagnosis, as well as on individualized treatment planning. According to guidelines, significant diagnostic components include 1) using DSM IV criteria [3] 2) collecting information about the child's symptoms in more than one setting; and 3) looking for coexisting conditions that may impede the diagnostic process or complicate treatment planning [1,2]. Stimulants are the most commonly used drug therapy in ADHD patients are burdened with a less than favorable pharmacokinetic and pharmacodynamic profile associated with their use [4-7]. In spite of the good response of many patients with ADHD to stimulant drugs, a substantial percent do not respond to or develop significant side effects from stimulants $[8,9]$. For this reason, new investigations have been initiated for ADHD treatment $[10,11]$. Deficiencies in certain vitamins and mineral have also been found to have some correlation with ADHD [12-14].

Zinc is an essential cofactor over 100 enzymes, both metalloenzymes and metal-enzyme complexes, required in the metabolism of carbohydrates, fatty acids, proteins, and nucleic acids $[12,13]$. Past studies have suggested that there may be a correlation of zinc deficiency and pathophysiology of ADHD [12-14]. Melatonin is a hormone that plays an important role in the regulation of dopamine, thought to be a factor in ADHD [14]. Melatonin is sold over the counter as dietary supplement and has been proven helpful in regulating the sleep cycle of children with ADHD. Zinc is involved in the production and modulation of melatonin, and therefore is also considered to be a factor in ADHD [12-14]. In this 6-week double blind, placebo controlled-trial, we assessed the effects of zinc plus methylphenidate vs. methylphenidate plus placebo in the treatment of children with attention deficit hyperactivity disorder. To our knowledge, this study is the first double blind and placebo controlled clinical trial assessing the adjunctive role of zinc in the ADHD.

\section{Methods \\ Trial organization}

This was a 6 week, parallel group, placebo controlled trial undertaken in Roozbeh Psychiatric Hospital, Tehran, Iran during January 2002-October 2003.

\section{Participants}

The subjects were 44 outpatient children (26 boys and 18 girls) between the ages of 5-11 (mean \pm SD was $7.88 \pm$ 1.67) who clearly met the DSM IV [3] diagnostic criteria for ADHD, and who were recruited from the outpatient child and adolescents clinic of Roozbeh Psychiatric Hospital. The diagnosis of ADHD was confirmed by a child and adolescent psychiatrist before participants were initiated into the study. All patients had combined subtype of ADHD and were newly diagnosed and had not received another stimulant medication prior to enrollment. Parents were carefully interviewed and asked to rate the severity of the DSM IV inattention symptoms their children displayed. Children were excluded if they had been previously diagnosed with a psychiatric disorder or mental retardation (I.Q. < 70). In addition, we excluded patients if they had a clinically significant chronic medical condition, including a past history of cardiovascular disease, organic brain disorder, seizures, current abuse or dependence on drugs within 6 months and current treatment with psychotropic medications. To participate, parents and children had to be willing to comply with all requirements of the study. After a description of the procedures and purpose of the study, written informed consent was obtained from each patient's parent or guardian. Informed consent (parent and children) was received before the administration of any study procedure or dispensing of study medication in accordance with the ethical standards of the investigative site's institutional review board and with the Helsinki declaration of 1975, as revised in 2000 [15].

\section{Study design}

Patients underwent a standard clinical assessment comprising a psychiatric evaluation, a structured diagnostic interview and a medical history. Patients were randomized to receive methylphenidate + zinc or methylphenidate + placebo in a 1: 1 ratio using a computergenerated code. The assignments were kept in sealed, opaque envelopes until the point of allocation. The randomization and allocation process was done by the pharmacist of the Roozbeh hospital. All study subjects were randomly assigned to receive treatment using either methylphenidate $1 \mathrm{mg} / \mathrm{kg} /$ day (bid, $7 \mathrm{AM}$ and $3 \mathrm{PM}$ ) + zinc sulfate $55 \mathrm{mg} /$ day (with approximately $15 \mathrm{mg}$ zinc element) (group 1) and methylphenidate $1 \mathrm{mg} / \mathrm{kg} /$ day (bid,

Table I: Baseline data

\begin{tabular}{ccc}
\hline & Zinc sulfate Group & Placebo Group \\
\hline Girl & 8 & 10 \\
Boy & 14 & 12 \\
Age (Mean \pm SD) & $8.04 \pm 1.73$ (year) & $7.73 \pm 1.63$ (year) \\
Ethnicity & All Persian & All Persian \\
\hline
\end{tabular}


Table 2: Clinical complications and side effects were reported as number per group.

\begin{tabular}{cccc}
\hline Side Effects & Zinc sulfate & Placebo & P \\
\hline Anxiety & 3 & 3 & Ns \\
Decreased Appetite & 8 & 7 & Ns \\
Difficulty Falling Sleep & 6 & 6 & Ns \\
Abdominal Pain & 8 & 4 & 0.31 \\
Nausea & 9 & 3 & 0.08 \\
Headache & 9 & 9 & Ns \\
Metallic taste & 13 & 0 & 0.0001 \\
\hline
\end{tabular}

$7 \mathrm{AM}$ and $3 \mathrm{PM}$ ) + Placebo (sucrose $55 \mathrm{mg}$ ) (group 2) for a 6 week double blind clinical trial. Throughout the study the person who administrated the medications, rater and patients were blind to assignments. The principal measure of the outcome was the Teacher and Parent ADHD Rating Scale [16] that has been used extensively in Iran in schoolage children, and provides valid measures of behavioral abnormality and attention. A score of at least 20 on the rating scales was required for entry into the study. Patients were assessed by a child psychiatrist at baseline, 14, 28 and 42 days after the medication started. 4 patients dropped out from the trial (two from each group), leaving 40 patients who completed the trial. Side effects were systematically recorded throughout the study and were assessed using a checklist administered by a resident of psychiatry on day $7,14,21,28$ and 42 (Table 2).

\section{Statistical analyses}

A two-way repeated measures analysis of variance (timetreatment interaction) was used. The two groups as a between-subjects factor (group) and the four measurements during treatment as the within-subjects factor (time) were considered. This was done for Teacher and Parent ADHD Rating Scale scores. In addition, a one-way repeated measures analysis of variance with a two-tailed post-hoc Tukey mean comparison test were performed on the change in Teacher and Parent ADHD Rating Scale scores from baseline. Results are presented as mean \pm SEM differences and were considered significant with $\mathrm{P}=0.05$. To compare the demographic data and frequency of side effects between the protocols, Fisher's exact test (two sided) was performed. A traditional "observed cases" (OC, the patients who completed the trial) analysis at 6 weeks was the primary efficacy analysis. In addition, intention to treat (ITT) analysis with the last observation carried forward (LOCF) procedure was also performed. All results discussed are based on OC analysis unless otherwise stated. To consider, $a=0.05, \beta=0.2$, the final difference between the two groups at least score of 5 on the Teacher and Parent ADHD Rating Scale, $\mathrm{S}=5$ and power $=80 \%$, the sample size was calculated at least 15 in each group.

\section{Results}

No significant differences were identified between patients randomly assigned to the group 1 or 2 conditions with regard to basic demographic data including age, gender and ethnicity (Table 1 ).

\section{Parent ADHD rating scale}

The mean \pm SEM scores of two groups of patients are shown in Fig. 1. There were no significant differences between two groups at day 0 (baseline) on the Parent ADHD Rating Scale $(P=0.86)$. Both groups showed a significant improvement over the 6 weeks of treatment $(\mathrm{P}<$ $0.000)$ and the trend was linear. The difference between the two treatments was significant as indicated by the effect of group, the between-subjects factor $(\mathrm{F}=4.15$, d.f. $=1, \mathrm{P}=0.048 \& \mathrm{~F}=4.27$, d.f. $=1, \mathrm{P}=0.045$ for $\mathrm{OC}$ and LOCF analyses respectively). The behavior of the two treatment groups was not homogeneous across the time (groups-by-time interaction, $\mathrm{P}<0.000$ ). In another word, the behavior of the two treatment groups changed across the trial. In addition, a one-way repeated measures analysis of variance showed a significant effect of both treatments on the Parent ADHD Rating Scale scores. In both groups post-hoc comparisons of the baseline the Parent ADHD Rating Scale scores with the scores at day 42 by means of the Tukey procedure revealed significant decreases from baseline. The differences between the two treatments were significant at the endpoint $(\mathrm{P}=0.0009$ \& $\mathrm{P}=0.001$ for OC and LOCF analyses respectively). A significant difference was observed on the reduction of scores of the Parent ADHD Rating Scale at week 6 compared to baseline in the two groups $(\mathrm{P}=0.0003 \& \mathrm{P}=$ 0.0001 for OC and LOCF analyses respectively).

\section{Teacher ADHD rating scale}

The mean \pm SEM scores of the two groups are shown in Fig. 2. No significant differences were observed at baseline on the Teacher ADHD Rating Scale $(P=0.85)$. Both groups showed a significant improvement over the 6 weeks of treatment $(\mathrm{P}<0.000)$ and the trend was linear. The difference between the two treatments was significant as indicated by the effect of the group, the between-sub- 


\section{Parent ADHD Rating Scale}

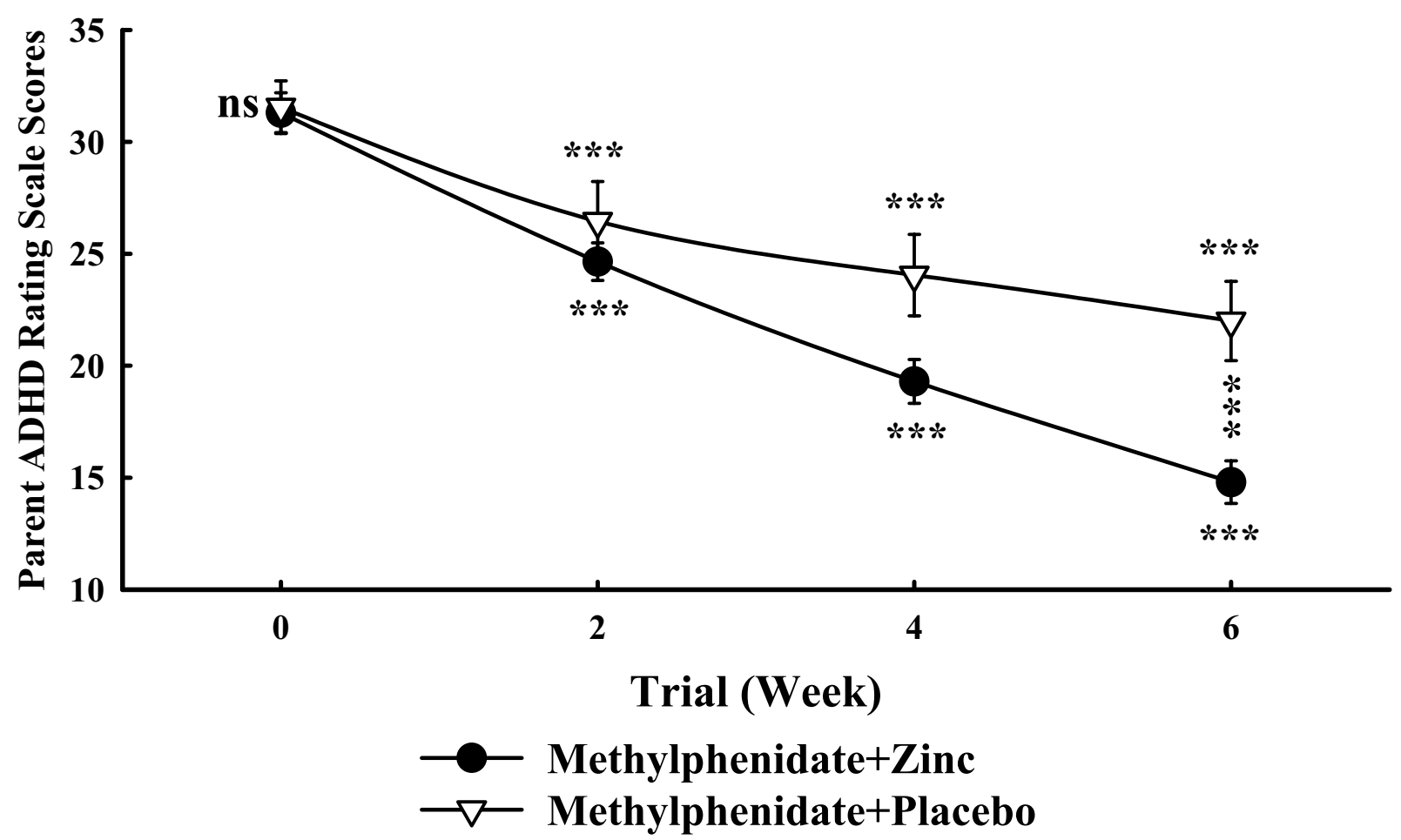

Figure I

Mean \pm SEM scores of two groups of children on the Parent ADHD Rating Scale. ns = non-significant, $* * *=P<0.00 \mathrm{I}$. The horizontal symbols $(* * *)$ were used to express statistical significance versus their respective baseline value and the vertical symbols are for between group comparisons.

jects factor $(\mathrm{F}=4.50$, d.f. $=1, \mathrm{P}=0.04 \& \mathrm{~F}=4.58$, d.f. $=1$, $\mathrm{P}=0.038$ for $\mathrm{OC}$ and $\mathrm{LOCF}$ analyses respectively). The behavior of the two treatment groups was not homogeneous across the time (groups-by-time interaction; $\mathrm{P}<0.000)$. In addition, a one-way repeated measures analysis of variance showed a significant effect of both treatments on the Teacher ADHD Rating Scale scores. In both groups post-hoc comparisons of the baseline the Teacher ADHD Rating Scale scores with the scores at day 42 by means of the Tukey procedure revealed significant decreases from baseline. The differences between the two treatments were significant at the endpoint $(\mathrm{P}=$ $0.0009 \& \mathrm{P}=0.001$ for OC and LOCF analyses respectively). A significant difference was observed on the reduc- tion of scores of the Teacher ADHD Rating Scale at week 6 compared to baseline in the two groups $(\mathrm{P}=0.0004 \& \mathrm{P}=$ 0.0005 for OC and LOCF analyses respectively).

\section{Retention in treatment}

In both groups the number of dropouts was 2 and this was lost in the follow up. Therefore, no significant difference was observed in the two groups.

\section{Clinical complications and side effects}

A number of probable side effects were studied (Table 2). Nausea was observed more in the zinc sulfate group. 


\section{Teacher ADHD Rating Scale}

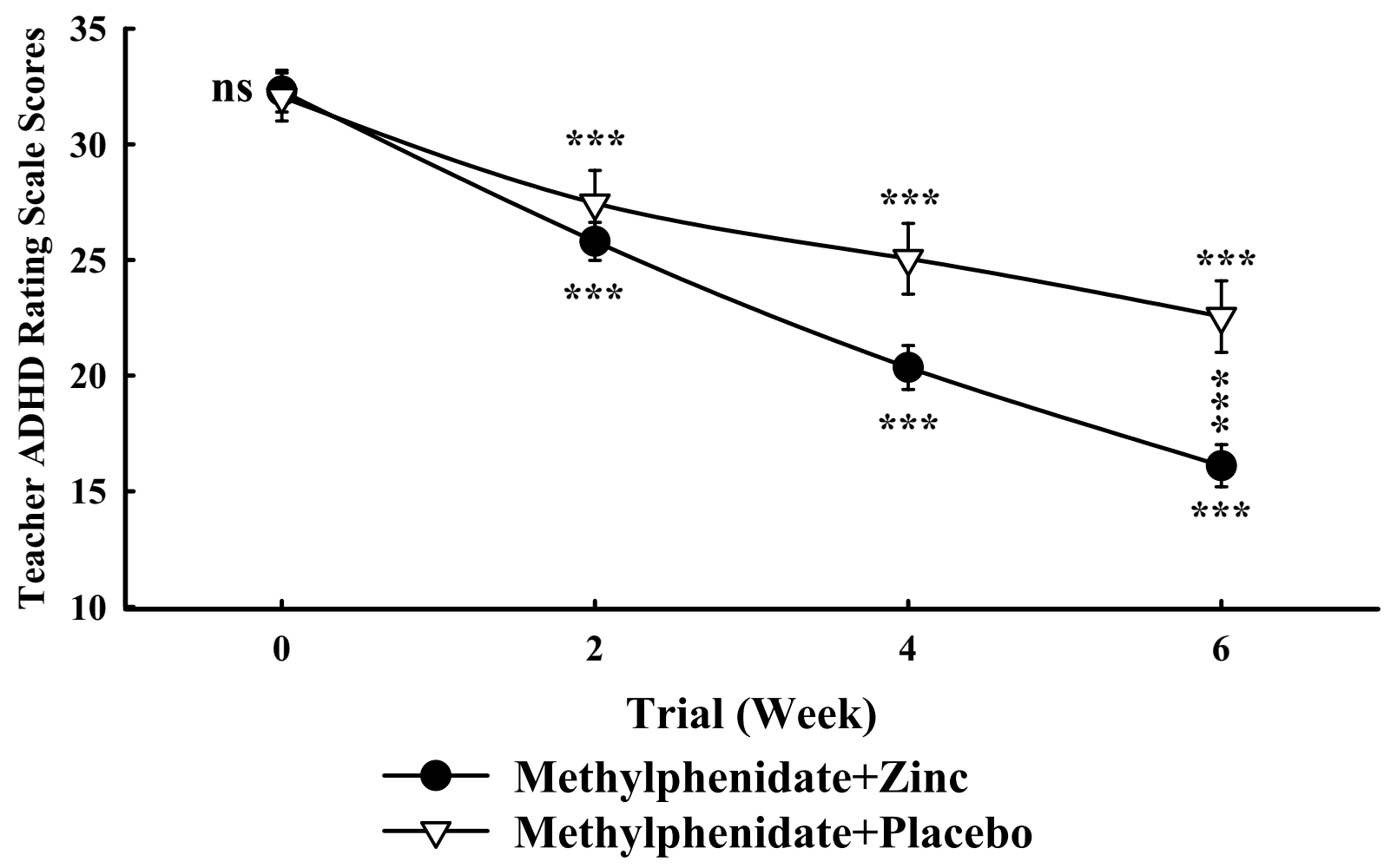

Figure 2

Mean \pm SEM scores of two groups of children on the Teacher ADHD Rating Scale. ns $=$ non-significant, $* * *=P<0.001$. The horizontal symbols $(* * *)$ were used to express statistical significance versus their respective baseline value and the vertical symbols are for between group comparisons.

\section{Discussion}

The diagnosis and management of children with ADHD remains a challenge [11]. In the last decade, several studies into the role of trace element in enlightening the aetiology of ADHD had been conducted. Most notable among the studies are the ones particularly examing the relation between zinc and ADHD [12-14]. Indeed, zinc is basic for the production and modulation of melatonin, which helps regulate dopamine function, supposed to be an important factor in ADHD and its treatment [14]. Therefore, ADHD children with zinc deficiency might benefit from a change in diet or from a therapeutic trial with zinc supplementation. The present study shows the Parent and Teacher Rating Scale scores improved with zinc sulfate over this 6-week, double blind and placebo controlled trial. The efficacy of zinc sulfate to obtain a better improvement in children with ADHD seems to support the role of zinc deficiency in the etiopathogenesis of ADHD $[12,13]$. The present study is in line with the recent trial that suggests a beneficial effect of zinc sulfate in the treatment of ADHD [17]. However, the present study assessed the adjunctive role of zinc in the ADHD for the first time. No significant differences were observed in the rates and reasons for discontinuation in treatment in the zinc and placebo groups. This finding suggested that tolerability of zinc and placebo was similar. Nausea was observed more in the zinc group but did not differ significantly between the two groups $(P=0.08)$. In addition, 
the metallic taste of zinc was complained of by the majority of cases in the zinc group (13 out of 22). The limitations of the present study, including lack of a full placebo group, using only a moderate dose of methylphenidate, the small number of participants, short period of follow up and lack of plasma zinc concentration should be considered so further research in this area is needed. However, a study from the Middle East region has reported that there may be a correlation of zinc deficiency and pathophysiology of ADHD [13] and suggests that the treatment of zinc in ADHD would be more beneficial in cases that have low zinc levels. In conclusion, this double-blind, placebo-controlled study demonstrated that zinc as a supplementary medication might be beneficial in the treatment of children with ADHD. However, further investigations and different doses of zinc are required to replicate these findings in children with ADHD.

\section{Competing interests}

None declared.

\section{Authors' contributions}

SA was the principal investigator and performed statistical analysis. MRM participated in the design of the study and was clinical coordinator. MK was the trialist. All authors read and approved the final manuscript.

\section{Acknowledgments}

This study was postgraduate thesis of Dr. M. Khademi. The authors thank Dr. M. Margaret Tegerizo for invaluable help.

\section{References}

I. Goldman L, Genel M, Bezman R, Slanetz P: Diagnosis and treatment of attention deficit/hyperactivity disorder in children and adolescents. JAMA 1998, 279: I 100-I 107

2. Mercuglioano $M$ : What is attention-deficit/hyperactivity disorder? Pediatr Clin North Am 1999, 46:83 I-43.

3. American Psychiatric Association: Diagnostic and Statistical Manual of Mental Disorders, (DSM-IV). American Psychiatric Association, Washington, D.C 41994.

4. Wilens TE, Biederman J: The stimulants. Psychiatr Clin North Am 1992, I 5: 191-222.

5. Gillberg C, Melander H, Knorring A: Long-term stimulant treatment of children with attention-deficit hyperactivity disorder symptoms. Arch Gen Psychiat 1997, 54:857-864.

6. Safer DJ: Central stimulant treatment of childhood attention deficit hyperactivity disorder. CNS Drugs 1997, 7:264-272.

7. Tenreiro K: Methylphenidate-placebo: A trial for attention deficit disorders. Int J Pharm Comp 200I, 5:21-22.

8. Klein RG, Landa B: Methylphenidate and growth in hyperactive children. Arch Gen Psychiat 1988, 45: I I27-II30.

9. Pataki CS, Carlson GA, Kelly KL, Rapport MD, Blancanlello TM: Side effects of methylphenidate and desipramine alone and in combination in children. J Am Acad Child Adolesc Psychiatry 1993, 32:1065-1072.

10. Prince JB, Wilens TE, Biederman J: A controlled study of nortriptyline in children and adolescents with attention deficit hyperactivity disorder. J Child \& Adolesc Psychopharmacol 2000, 10:193-204.

II. Kehpe WA: Treatment of attention deficit hyperactivity disorder in children. The Ann Pharmacother 2001, 35:1 I30-I I34.

12. Toren P, Elder S, Sela BA, Wolmer L, Weitz W, Inbar D, Koren S, Reiss A, Weizman R, Laor N: Zinc deficiency in attention deficit hyperactivity disorder. Biol Psychiat 1996, 40:1308-1310.
13. Bekarglu M, Aslan Y, Gedik Y, Deger O, Mocan H, Erduran E, Karahan $C$ : Relation between serum free fatty acids and zinc, and attention deficit hyperactivity disorder: A research note. J Child Psyhol Psychiat 1996, 37:225-227.

14. Kirby K, Floriani V, Bernstein H: Diagnosis and management of attention-deficit hyperactivity disorder in children. Cur Opinion in Pediatr 200 I, 13:190-199.

15. World Medical Association Declaration of Helsinki: Ethical principles for medical research involving human subjects. 2000 [http://www.wma.net].

16. Dupaul GP: Parent and teacher ratings of ADHD symptoms: Psychometric properties in a community based sample. J Clin Child Psychol 1991, 20:245-253.

17. Bilici M, Yildirim F, Kandil S, Berkaroglu M, Yildirmis S, Deger O, Ulgen M, Yildiran A, Aksu H: Double blind, placebo-controlled study of zinc sulfate in the treatment of attention deficit hyperactivity disorder. Prog in Neuropsychopharm \& Biol Psychiat 2004, 28: $181-190$.

\section{Pre-publication history}

The pre-publication history for this paper can be accessed here:

http://www.biomedcentral.com/1471-244X/4/9/prepub
Publish with Biomed Central and every scientist can read your work free of charge

"BioMed Central will be the most significant development for disseminating the results of biomedical research in our lifetime. " Sir Paul Nurse, Cancer Research UK

Your research papers will be:

- available free of charge to the entire biomedical community

- peer reviewed and published immediately upon acceptance

- cited in PubMed and archived on PubMed Central

- yours - you keep the copyright

Submit your manuscript here:

http://www.biomedcentral.com/info/publishing_adv.asp
BiolMedcentral 